\title{
Control and theoretical modeling of the growth process of AlN six-fold and multifold armed dendritic crystals
}

Hayk H. Nersisyan ${ }^{a, b}$, Wan Bae Kim ${ }^{a}$, Seong Hun Lee ${ }^{b}$, Bung Uk Yoo ${ }^{c}$,Hyuck Choi ${ }^{d}$, Hyun You $\mathrm{Kim}^{d}$, Jong Hyeon Lee $\mathrm{a}^{a, b * *}$

aRASOM, Chungnam National University, 99 Daehak-ro, Yuseong-gu, Daejeon 34134, Republic of Korea

${ }^{\mathrm{b}}$ Graduate School of Materials Science and Engineering, Chungnam National University, 99 Daehak-ro, Yuseong-gu, Daejeon 34134, Republic of Korea

${ }^{c}$ Graduate School of Energy Science and Technology, Chungnam National University, 99 Daehakro, Yuseong-gu, Daejeon 34134, Republic of Korea

dDepartment of Materials Science and Engineering, Chungnam National University, 99 Daehakro, Yuseong-gu, Daejeon 34134, Republic of Korea

*Corresponding Author: Jong Hyeon Lee (E-mail address: jonglee@cnu.ac.kr)

\section{(Supporting Information)}




\section{EXPERIMENTAL SECTION}

\section{Combustion experiments}

A paper cup was used in the experiment as the cup becomes charred in the combustion process and assisted in the easy filtration of nitrogen gas from the surroundings to the combustion wave. The main combustion experiments were conducted under 4.0 MPa nitrogen pressure, as this was the most favorable pressure level for obtaining large amounts of dendritic crystals according to preliminary experiments conducted in the 1.0-5.0 MPa pressure range. In order to increase temperature measurement accuracy, the thermocouples were preliminary coated with a thin layer of AlN.

\section{RESULTS AND DISCUSSION}

The SEM micrographs of the Al particles before and after heat treatment are shown in Figure S1a and S1b. As seen in the images, the size of the initial Al particles was in the 10-70 $\mu \mathrm{m}$ range (mean size $\sim 40 \mu \mathrm{m}$ ) and the particles were mainly globular in shape with a small amount of spherical particles (Figure S1a). The addition of $\mathrm{Al}_{2} \mathrm{O}_{3}$ was effective in protecting the molten particles from the coagulation process, resulting in more spherical particles, as shown in Figure S1b. 


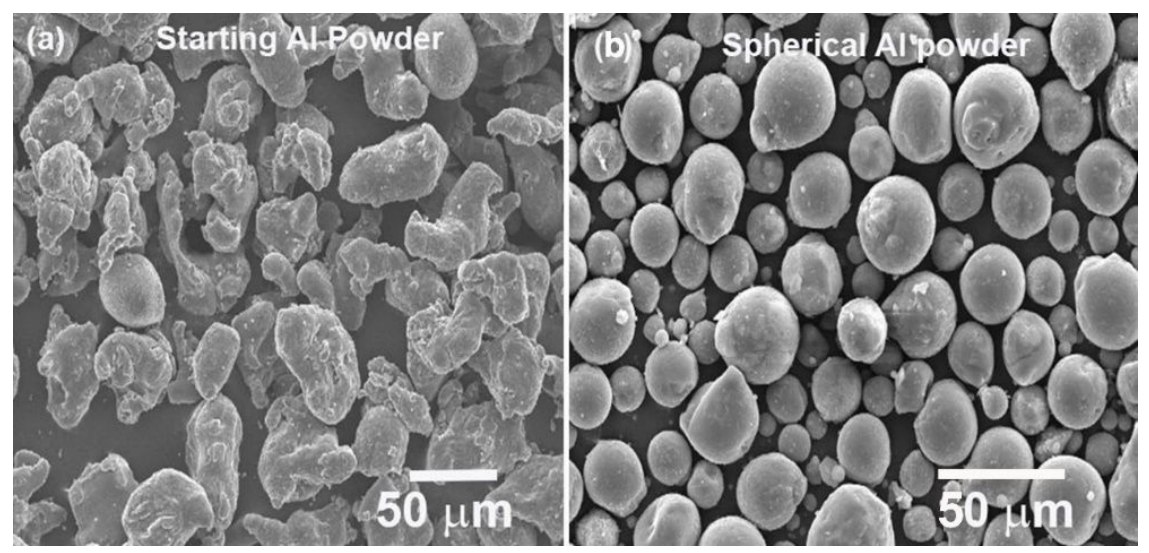

Figure S1. SEM micrographs of aluminum powders: (a) initial Al powder, (b) Al powder heat treated at $1000{ }^{\circ} \mathrm{C}$ with $\mathrm{Al}_{2} \mathrm{O}_{3}$.

\section{SEM morphologies}

Figure S2 shows additional micrographs of AlN dendritic crystals obtained at the $k=0-0.3$ interval. It is clear that $\mathrm{Al}_{2} \mathrm{O}_{3}$ assisted in producing dendritic crystals that are uniform in shape. In addition, the shape of the crystals is more simplified with increasing amounts of $\mathrm{Al}_{2} \mathrm{O}_{3}$. 

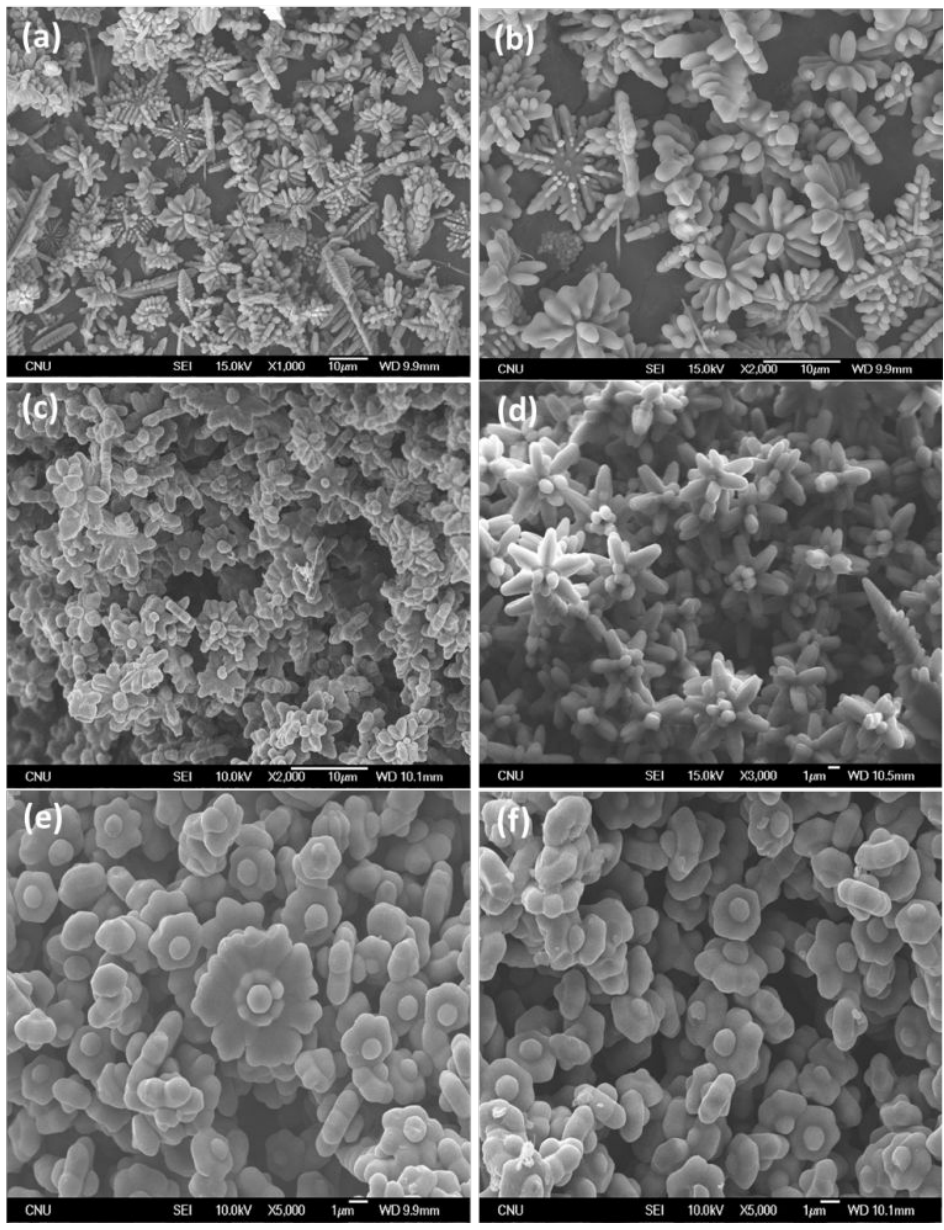

Figure S2. SEM images of six-fold symmetry crystals prepared from the $\mathrm{Al}+0.1 \mathrm{AlF}_{3}+k \mathrm{Al}_{2} \mathrm{O}_{3}$ mixture: (a, b) $k=0$;(c) $k=0.05$; (d) $k=0.2$; (e, f) $k=0.3$.

$\mathrm{C} 0$

The large feather-like dendritic fragments obtained at $k=0$ and the corresponding morphology details are shown in Figure S3. The dendrites have a central trunk or central pit and primary (1), secondary (2), and tertiary dendritic arms (3). The length of the dendrites varies from fifty to several hundreds of micrometers. In addition to feather-type dendrites, an abundance of six-fold and multifold symmetry dendrites were produced at $k=0$. 

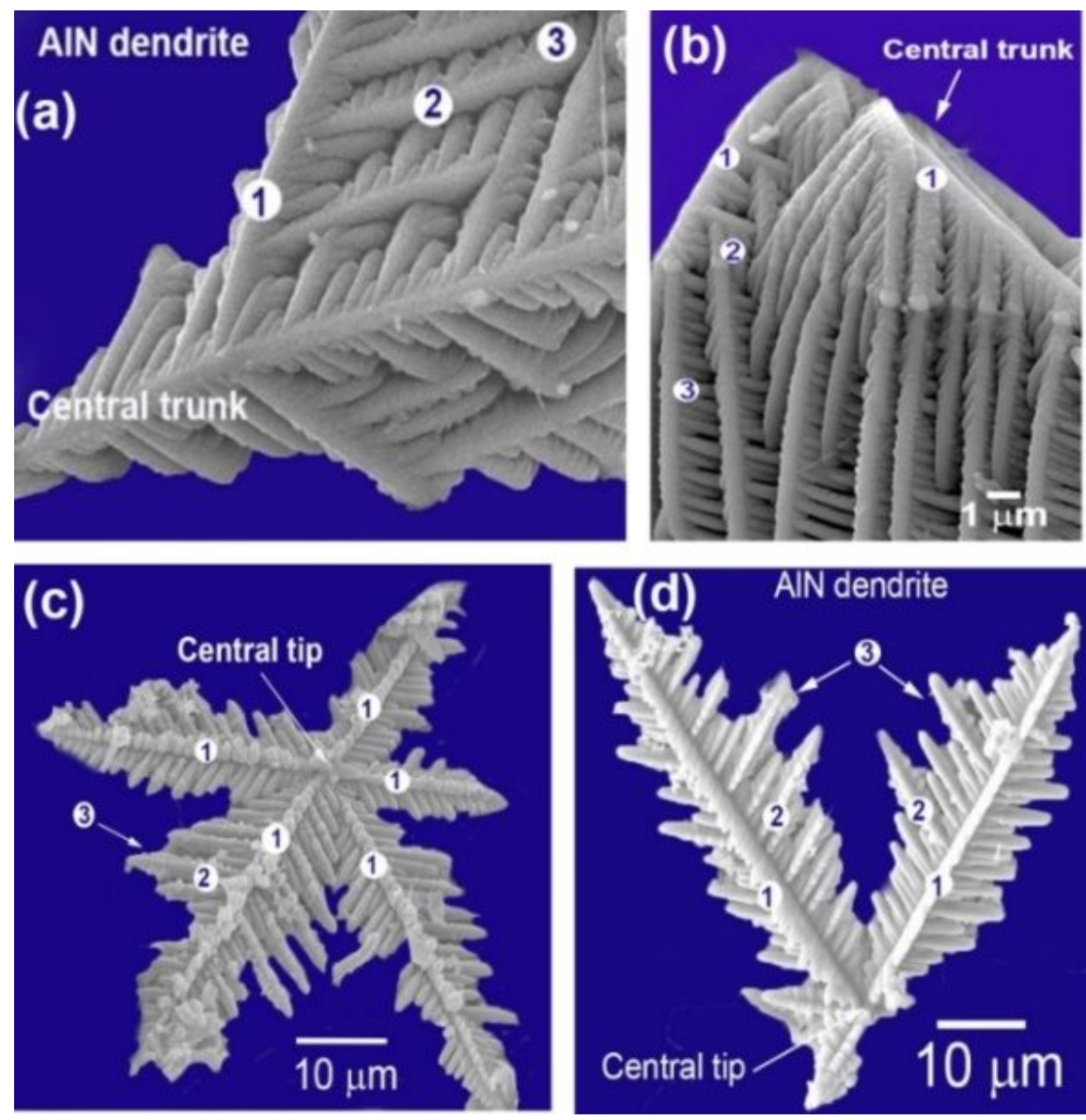

Figure S3. Feather-like dendrites of AlN: (1) central trunk (or pit), (2) primary dendritic arms; (2) secondary dendritic arms; (3) tertiary dendritic arms.

\section{DFT calculation}

Table S1. Quality test results of the DFT calculation

\begin{tabular}{|c|c|c|c|}
\hline Surface index & k-point grid & $\begin{array}{c}\text { Electron } \\
\text { energy cutoff }(\mathrm{eV})\end{array}$ & $\begin{array}{c}\text { Surface energy } \\
\left(\mathrm{J} / \mathrm{m}^{2}\right) \\
\end{array}$ \\
\hline
\end{tabular}




\begin{tabular}{llll}
\hline & $2 \times 2 \times 1$ & 400 & 3.49 \\
$(0 \overline{1} 11)$ & $4 \times 4 \times 1$ & 400 & 3.50 \\
& $2 \times 2 \times 1$ & 520 & 3.49 \\
\hline & $2 \times 2 \times 1$ & 400 & 3.54 \\
$(10 \overline{1} 1)$ & $4 \times 4 \times 1$ & 400 & 3.54 \\
& $2 \times 2 \times 1$ & 520 & 3.57 \\
\hline \hline
\end{tabular}

Phase-field calculation. For dendrite shape evaluation, a two-dimensional (2D) space was considered and the PF interface equation was utilized by taking the functional derivative of Ginzburg-Landau free energy as follows: ${ }^{29,30}$

$\frac{\partial \phi}{\partial \mathrm{t}}=\frac{\partial}{\partial \mathrm{y}}\left(\varepsilon \frac{\partial \varepsilon \partial \phi}{\partial \theta \partial \mathrm{x}}\right)-\frac{\partial}{\partial \mathrm{x}}\left(\varepsilon \frac{\partial \varepsilon \partial \phi}{\partial \theta \partial \mathrm{y}}\right)+\nabla \cdot\left(\varepsilon^{2} \nabla \phi\right)+\phi(1-\phi)\left(\phi-\frac{1}{2}+m\right)$

Here, $\phi$ is a phase-field parameter that satisfies the criteria required to ensure that the phase-field takes on fixed values (' 1 ' for a solid and ' 0 ' for a liquid), $\boldsymbol{x}$ and $\boldsymbol{y}$ are the spatial positions, $\mathbf{t}$ is time, $\theta$ is the angle between the growth vector and a certain direction, $\boldsymbol{\varepsilon}$ is the anisotropic gradient energy coefficient that determines the thickness of the interface layer, and $\boldsymbol{m}$ is the driving force for the interface motion proportional to supercooling. In order to take anisotropy into account, $\varepsilon$ is specified as follows:

$\boldsymbol{\varepsilon}=1+\delta \cos \left(\mathrm{j}\left(\theta-\theta_{\mathrm{o}}\right)\right.$

The parameter $\boldsymbol{\delta}$ is the strength of anisotropy and $\mathbf{j}$ is a mode number of anisotropy.

The evolution of temperature field, $\mathrm{T}$, was derived from the conservation law of enthalpy as follows: ${ }^{20,21}$

$$
\frac{\partial \mathrm{T}}{\partial \mathrm{t}}=\nabla^{2} \mathrm{~T}+k \frac{\partial \phi}{\partial \mathrm{t}}
$$


Where $\mathbf{T}$ is non-dimensional in this calculation to have the characteristic cooling temperature as ' 0 ' and the equilibrium temperature as ' 1 '. Here, $\boldsymbol{k}$ is dimensionless latent heat, which is proportional to latent heat and inversely proportional to the strength of cooling.

Figure S4 shows the growth of a single seed in a square cell depending on the number of steps for an anisotropy value of 12 . In the case of a single seed at the center of the calculation domain, the crystal exhibited 12 dendrite arms with secondary branches after 1000 steps. The dendritic arms and secondary branches continued to grow in size as the number of steps increased. After 3000 steps, the shape of the dendrite became significantly deformed as the dendritic arms became relatively thick. The branching of the dendritic arms began at 5,000 steps, and a well-branched structure with 12 dendritic arms was obtained after 20,000 steps.

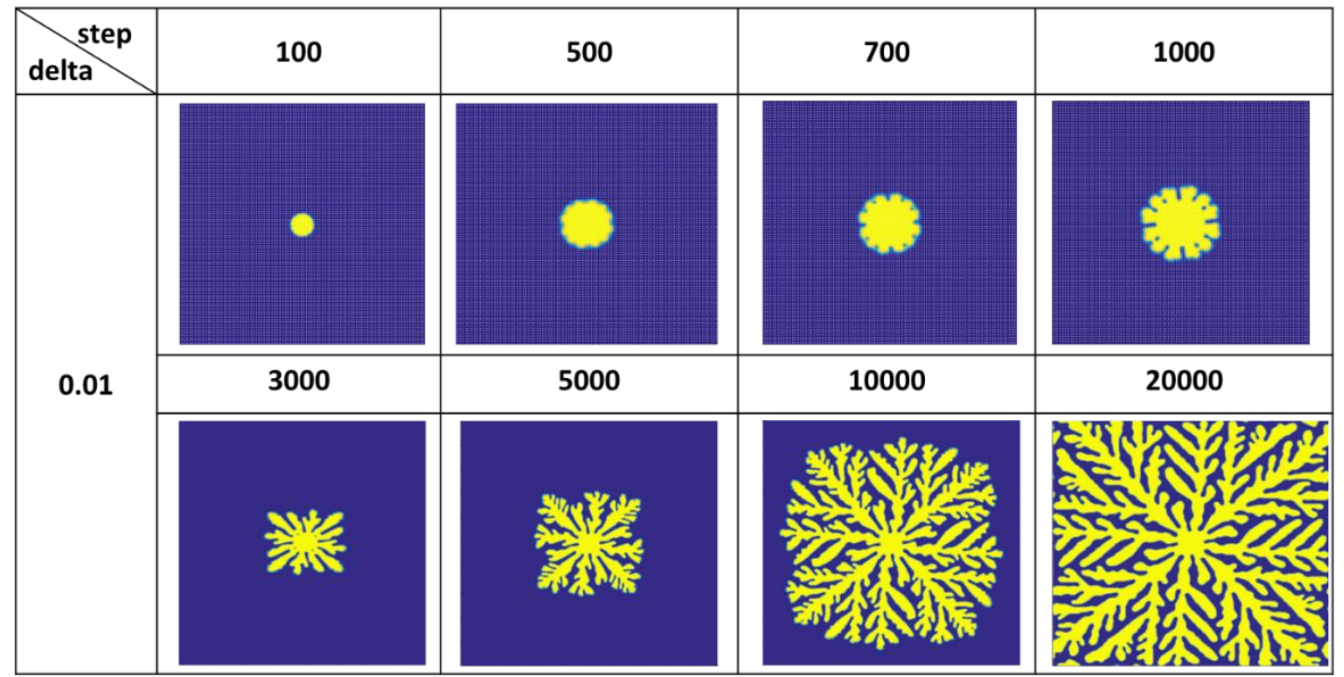

Figure S4. Initial nucleation conditions and dendritic crystal growth towards a supercooled melt with an anisotropy value of 12 .

The phase-field calculation made for an anisotropy value of 12 is shown in Figure S5 


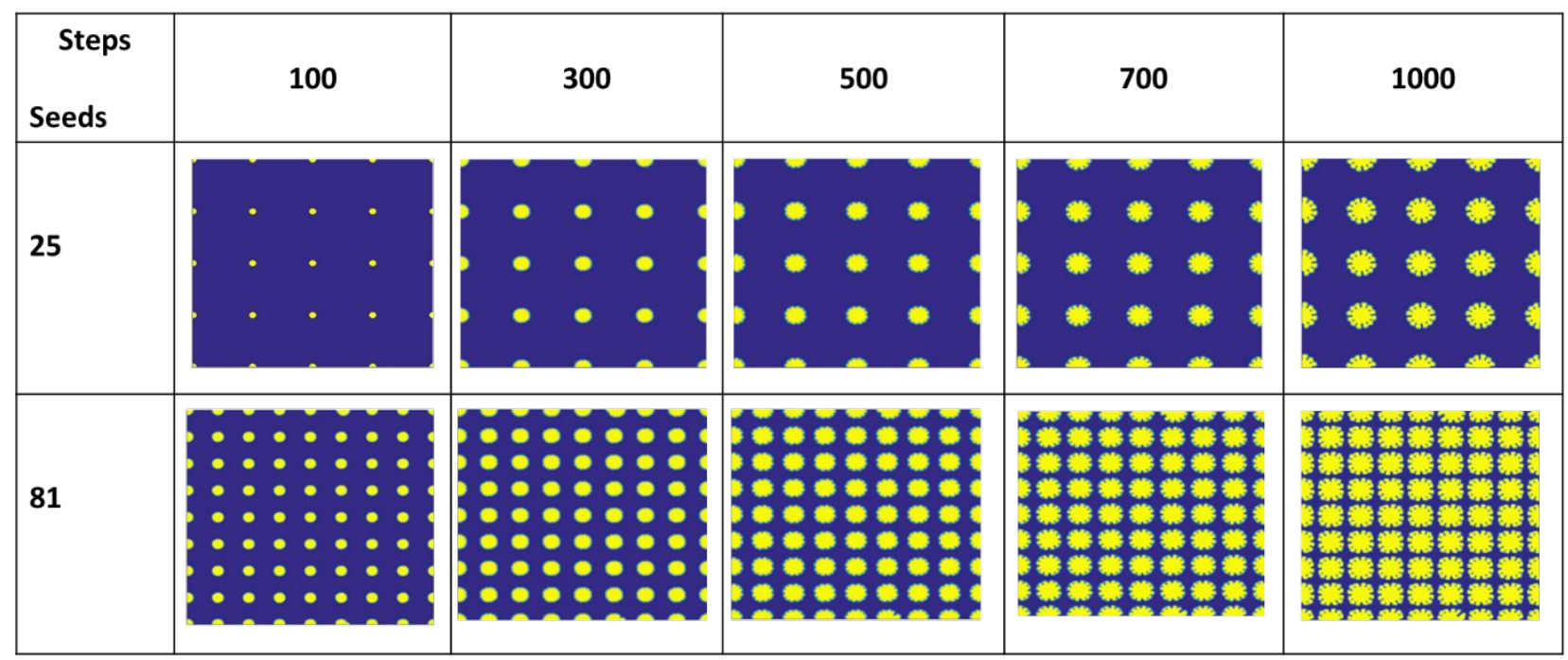

Figure S5. Initial nucleation conditions and dendritic crystal growth towards a supercooled melt for different crystal densities (anisotropy 12). 